Journal of Fundamental and Applied Sciences

ISSN 1112-9867

Available online at

http://www.jfas.info

\title{
APPLICATION OF FUZZY ENSEMBLES FOR OPTIMAL DISTRIBUTION OF POWER IN ELECTRICAL NETWORKS
}

\author{
Department of Electrotechnics, Faculty of Electrical Engineering, El-Oued University \\ Center, Algeria.
}

A. Guediri* and D. Ben Attous

Received: 03 February 2011 / Accepted: 05 June 2010 / Published online: 30 June 2011

\begin{abstract}
Optimal power flow calculation (OPF), used to optimize specific aspects of power system operations, usually employ standard mathematical programming techniques. These techniques are not suitable to handle many practical considerations encountered in power systems, including the uncertainty of the operational constraints [1].

They can be relaxed temporarily, if necessary, to obtain feasible solutions. For taking well into account this type of constraints, one proposes in this work the application of a method based on fuzzy sets to the OPF problem. The developed method has been tested on standard scale power systems (IEEE30bus).
\end{abstract}

Keywords: optimal power flow, Fuzzy optimization, Fuzzy mathematical programming

\section{INTRODUCTION}

Nous avons assisté ces dernières années à une croissance très rapide des travaux utilisant la logique floue dans les systèmes électriques. Cela est dû à la simplicité de leurs mécanismes, la facilité de leur mise en application et leur efficacité même pour les problèmes complexes. Nous appliquons ici l'algorithme d'FOPF étape par étape à l'optimisation de l'écoulement de puissance sur un simple réseau avec des contraintes souples sur la puissance transportée par lignes.

Author Correspondence, e-mail:kkarim_elect@yahoo.fr

ICID: 1020765 


\section{FORMULATION DU PROBLEME DE L'OPF PAR LES ENSEMBLES FLOUS}

Comme mentionné plus haut, le problème de l'OPF possède plusieurs caractéristiques floues.

Le forcement des contraintes souples n'a pas besoin d'être exact à 100\%, d'autre part la minimisation de la fonction objective ne doit pas aussi être rigide. Par conséquent, la théorie des ensembles flous peut aisément être appliquée au problème de l'OPF afin de modéliser ces considérations pratiques avec exactitude.

Le problème de l'OPF peut être formulé par la fonction objective et contraintes floues, comme suit [2] [3]

$$
\begin{aligned}
& \text { Minimiser } \quad f(x) \simeq C_{0} \\
& \text { sujet } \quad \grave{a} g_{j}(x)=0 \quad, \quad j=1, \ldots, n \text {. } \\
& h_{i}(x) \tilde{\leq} d_{i} \quad i=1, \ldots m .
\end{aligned}
$$

Où, $\widetilde{\leq}$ désigne une inégalité floue.

L'équation (I-1) montre que l'objectif est de minimiser $\mathrm{f}(\mathrm{x})$, de telle manière qu'elle n'excède pas la valeur $C_{0}$ "beaucoup trop". L'équation (I-3) montre que la solution doit satisfaire la contrainte $h_{i}(x)$ le plus possible, et aussi ne pas dépasser la limite $d_{i} \quad$ "beaucoup trop".

L'équation (I-2) représente des contraintes d'égalités conventionnelles (rigides), qui doivent être satisfaites exactement. Donc, l'inégalité floue $\tilde{\leq}$ a été utilisée pour exprimer la fonction objective et les contraintes floues ensemble.

La fonction d'appartenance de l'inégalité floue $\widetilde{\leq}$, peut être représentée par une fonction trapézoïdale (Fig. I-1), donnée par

$$
\mu_{f}(f(x))=\left\{\begin{array}{lr}
1 & f(x) \leq C_{0} \\
\left(C_{0}+\delta_{c 0}-f(x)\right) / \delta_{c 0} & C_{0} \prec f(x) \prec C_{0}+\delta_{c 0} \\
0 & f(x) \succ C_{0}+\delta_{C 0}
\end{array}\right.
$$


Cette fonction d'appartenance décroît linéairement, quand le coût $f(x)$ se situe entre $C_{0}$ et $C_{0}+\delta_{c 0}$. Le coût $C_{0}+\delta_{c 0}$ dans l'équation (I-4) constitue le coût maximal admissible.

Cette valeur est souvent égale au coût de fonctionnement donné par la solution de l'écoulement de puissance, qui représente l'état initial de fonctionnement non optimisé.

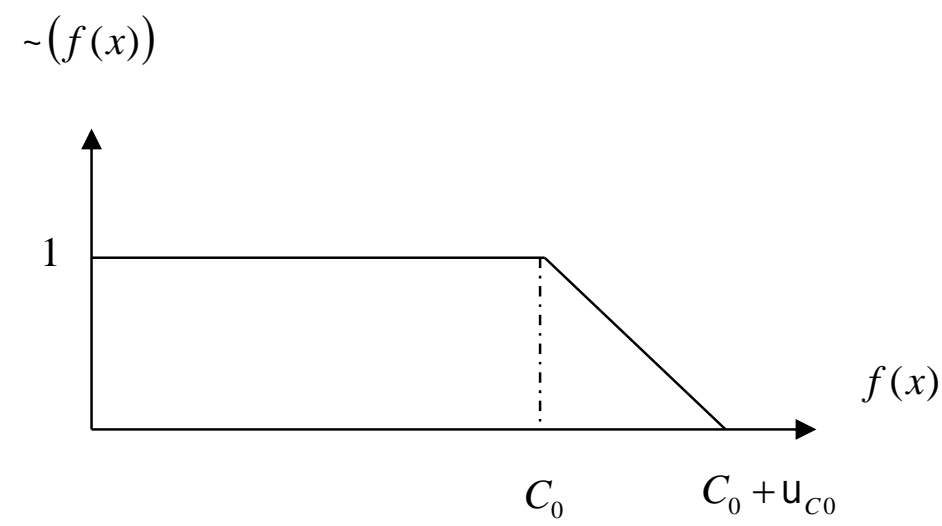

Fig.1. Fonctions d'appartenance de la fonction objective.

Donc, le coût le plus bas $C_{0}$ est déterminé par l'utilisateur désirant une réduction maximale $\delta_{c 0}$ du coût de fonctionnement. Le choix de ces paramètres reste subordonné à certaines considérations pratiques spécifiques au fonctionnement du système de puissance.

De la même manière, les fonctions d'appartenance des contraintes floues, sont similaires à celle de la fonction objective:

${ }^{\mu} h i\left(h_{i}(x)\right)=\left\{\begin{array}{lr}1 & h_{i}(x) \leq d_{i} \\ \left(d_{i}+\delta_{d i}-h_{i}(x)\right) / \delta_{d i} & d_{i} \prec h_{i}(x) \leq d_{i}+\delta_{d i} \\ 0 & h_{i}(x) \succ d_{i}+\delta_{d i}\end{array}\right.$

$d_{i}$ est la limite minimale désirée, et $d_{i}+\delta_{d i}$ est la limite maximum autorisée.

Physiquement, pour les contraintes souples, $d_{i}$ représente la limite normale de fonctionnement, et $d_{i}+\delta_{d i}$ est la limite d'urgence (critique). Il est autorisé de dépasser "un petit peu" la limite normale d'une contrainte souple, mais la limite d'urgence ne doit jamais être dépassée.

Pour les contraintes rigides, la fonction d'appartenance est donnée par 


$$
\mu_{h i}\left(h_{i}(x)\right)= \begin{cases}1 & h_{i}(x) \leq d_{i} \\ 0 & h_{i}(x) \succ d_{i}\end{cases}
$$

\section{Méthodologie de solution}

La solution du problème de l'OPF formulé par les équations (I-1), (I-2) et consiste à minimiser la fonction objective floue, avec la satisfaction des contraintes floues et non floues [4].

Le degré de satisfaction du coût et des contraintes floues, peut être représenté par une seule fonction d'appartenance $\mu_{D}(x)$, définie par:

$\mu_{D}(x)=\mu_{f}(f(x))$. et $\mu_{h 1}\left(h_{1}(x)\right)$.et. $\mu_{h 2}\left(h_{2}(x)\right)$ et .....et. $\mu_{h m}\left(h_{m}(x)\right)$

La fonction d'appartenance $\mu_{D}(x)$ est définie par le minimum de toutes les fonctions d'appartenance de la fonction coût et des contraintes

$\mu_{D}(x)=\min \left\{\mu_{f}(f(x)), \mu_{h 1}\left(h_{1}(x)\right), \mu_{h 2}\left(h_{2}(x)\right), \ldots \ldots \mu_{h m}\left(h_{m}(x)\right)\right\}$

Pour passer du domaine des ensembles flous au domaine ordinaire, la fonction d'appartenance $\mu_{D}(x)$ Doit être défuzzifiée par une méthode choisie.

Si on choisit la méthode de défuzzification du maximum, le problème de l'OPF revient à chercher le maximum de $\mu_{D}(x)$ en fonction de $\mathrm{x}$.

Si on pose $z=\mu_{D}(x)$, on peut écrire

$$
\max \left(\mu_{D}(x)\right)=\max _{z \in[0,1], x}\left\{\min \left\{\begin{array}{l}
\mu_{f}(f(x)), \mu_{h 1}\left(h_{1}(x)\right), \\
\mu_{h 2}\left(h_{2}(x)\right) \ldots \ldots, \mu_{h m}\left(h_{m}(x)\right)
\end{array}\right\}\right\}
$$

L'expression (I-5) peut être réécrite sous la forme conventionnelle suivante

$$
\begin{gathered}
\text { Maximiser } \quad z \\
\text { Sujet } \grave{a} \quad z \leq \mu_{f}(f(x)) \\
z \leq \mu_{h i}\left(h_{i}(x)\right), \quad i=1, \ldots, m \\
0 \leq z \leq 1
\end{gathered}
$$


En substituant les fonctions d'appartenance (I-3) et (I-4) dans les expressions (I10) et (I-1), la formulation floue du problème de l'OPF peut être convertie en un nouveau problème d'optimisation conventionnel, donné par

$$
\begin{aligned}
& \text { Minimiser }(-z) \\
& \text { sujet à } \quad f(x)+\delta_{c 0} z \leq C_{0}+\delta_{c 0} \\
& \quad h_{i}(x)+\delta_{d i} z \leq d_{i}+\delta_{d i} \\
& \quad i=1, \ldots, m \\
& 0 \leq z \leq 1
\end{aligned}
$$

L'introduction d'une contrainte de coût ainsi qu'une nouvelle variable z, représentant le degré de satisfaction.

L'objectif devient: maximiser la fonction d'appartenance variable (ou minimiser $-\mathrm{z}$ ).

Il faut mentionner que si $\delta_{d i}$ est nul, l'inégalité (I-3) est équivalente à une contrainte rigide (ordinaire).

Remarque

Afin de rendre la nouvelle formulation floue, équivalente à la formulation conventionnelle, lorsque la solution est admissible $(z=1)$, la fonction objective peut être modifiée comme suit [5]

$\mathrm{M}$ inimiser $\quad(-z+k f(x))$

$\mathrm{K}$ est un petit nombre positif, tel que:

k. $\mathrm{f}(\mathrm{x})<<1$.

$\checkmark \quad \mathrm{Si} \mathrm{z}=1$, l'OPF flou est équivalent à l'OPF conventionnel qui minimise la fonction $f(x)$ et satisfait les contraintes.

$\checkmark$ Si la solution se trouve dans la région floue (c a d que quelques contraintes sont légèrement violées), le premier terme de la fonction objective, -z, domine le processus d'optimisation et le problème est approximativement équivalent à celui de l'OPF flou.

\section{Algorithme de l'OPF flou}

Pour la résolution du nouveau problème d'optimisation, formulé par (I-1), (I-2) et (I-3). Les étapes de base de l'algorithme utilisé se résument par:

Etape (1): Résoudre le problème de l'écoulement de puissance, pour obtenir une solution acceptable qui satisfait les contraintes d'égalité. 
Etape (2): Linéariser les contraintes floues données par (I-10) et (I-11), autour de la solution de l'écoulement de puissance.

Etape (3) : Résoudre le problème de programmation linéaire et obtenir les accroissements optimaux des variables de contrôle $\Delta x$ et de la variable floue ${ }^{\Delta z}$.

Etape (4): Mettre à jour les variables : $x^{k+1}=x^{k}+\Delta x$ et $z^{k+1}=z^{k}+\Delta z$.

Etape (5): Obtenir une nouvelle solution de l'écoulement de puissance, en utilisant les nouvelles variables de contrôle.

Etape (6) : $\mathrm{Si}^{\Delta x}$ et $\Delta z$ de l'étape (3), sont inférieures à une certaine tolérance, le processus converge, sinon, aller à l'étape (2).

Dans les étapes (1) et (5) on a utilisé la méthode découplée rapide, pour résoudre les équations non linéaires de l'écoulement de puissance $g_{i}(x)=0$. La solution de l'écoulement de puissance est nécessaire puisque celle-ci nous donne le point de fonctionnement initial ${ }^{x_{0}}$ et le point suivant (actualisé) $x^{k+1}$, ainsi que tous les autres points résultants du problème d'optimisation non linéaire original.

Dans l'étape (2), les contraintes d'inégalités non linéaires, sont linéarisées autour $\mathrm{de}^{x^{k}}$ et $z^{k}$, comme suit $J_{f}\left(x^{k}\right) . \Delta x+\delta_{c 0} \Delta z \leq C_{0}+\delta_{c 0}-f\left(x^{k}\right)-\delta_{c 0} z^{k} \quad \begin{gathered}J_{h i}\left(x^{k}\right) \cdot \Delta x+\delta_{d i} \Delta z \leq d_{i}+\delta_{d i}-h_{i}\left(x^{k}\right)-\delta_{d i} z^{k} \\ i=1, \ldots, m\end{gathered}$

17)

$J_{f}\left(x^{k}\right)$ Et ${ }^{J_{h i}}\left(x^{k}\right)$ i sont respectivement, les vecteurs et matrices jacobéennes de $\mathrm{f}(\mathrm{x})$ et $h_{i}(x)$.

5. Défuzzification des ensembles flous

Généralement, la solution d'un problème utilisant les ensembles flous, est un résultat exprimé en termes de valeurs floues (fonctions d'appartenance).D'autre part, pour exploiter ou appliquer physiquement ces résultats, on doit utiliser des valeurs ordinaires.

Cette operation qui consiste à convertir les valeurs floues en valeurs ordinaires, s'appelle la défuzzification.

La méthode de défuzzification la plus utilisée est certainement, la méthode du centre de gravité, dont le principe est de prendre l'abscisse du centre de gravité de la surface de la 
fonction d'appartenance résultante. Cependant, cette méthode de défuzzification est parfois complexe et demande des calculs importants. C'est pour cette raison qu'on utilise souvent une autre méthode plus simple qui est la méthode du maximum, dont le principe est de prendre l'abscisse du maximum de la fonction d'appartenance résultante [6].

\section{Test de l'algorithme FOPF avec des contraintes souples sur les puissances transportées par les lignes.}

Dans cette partie, on va appliquer l'algorithme d'optimisation de l'écoulement de puissance par les ensembles flous FOPF sur le réseau 30 jeux de barres (IEEE 30-bus). L'algorithme FOPF va résoudre le problème en prend en compte le coût de production ainsi que les limites de puissances actives transmises par les lignes de transport, seront prises comme contraintes floues. Le reste des contraintes d'égalité et d'inégalité seront modélisées par des contraintes rigides (non floues).

Les fonctions d'appartenances des contraintes floues, sont linéairement décroissantes entre la valeur normale et la valeur maximale admissible (limite critique). La fonction d'appartenance sera égale à 1 quand la contrainte est au dessous de la limite normale, et égale à 0 lorsque la contrainte est au dessus de la limite maximale admissible.

\section{Réseau test IEEE 30-bus:}

\section{FOPF en tenant compte le surcharge des lignes}

\section{CAS $\mathrm{N}^{\circ} 1$}

Le coût non optimisé de l'écoulement de puissance (NR_PF) est 901 \$/h qui sera pris comme le coût maximal admissible $C_{0}+\delta_{c 0}$. Le coût désiré $C_{0}$ doit avoir une valeur inférieure au coût non optimisé, et supérieure au coût optimal conventionnel. La valeur de $C_{0}$ sera choisit égal à $890 \$ / \mathrm{h}$.

Les résultats obtenus sont comme suit:

$$
C_{0}=890.00 \$ / \mathrm{h}, \delta_{c 0}=11.00 \$ / \mathrm{h} .
$$

Solution $\mathrm{z}=1.00$, Coût $=859.36 \$ / \mathrm{h}$, Pertes $=5.32 \mathrm{MW}$.

Les contraintes forcées des puissances actives transmises, sont montrées dans le tableau I-1. Les résultants obtenus sont identiques à ceux de l'OPF conventionnel. 
Tableau I-1. Puissance actives transmises forcées du réseau électrique à 30 J.B. - cas $\mathrm{n}^{\circ} 1$

\begin{tabular}{lllll}
\hline$N^{0}$ Ligne & $\begin{array}{l}\text { Dub } \\
\text { Vers j-b }\end{array}$ & $\begin{array}{l}\text { Limite } \\
\text { normale } \\
(\mathrm{MW})\end{array}$ & $\begin{array}{l}\text { Limite } \\
\text { d'urgence } \\
(\mathrm{MW})\end{array}$ & $\begin{array}{l}\text { Puissance } \\
\text { active (MW) }\end{array}$ \\
\hline 1 & $1-2$ & 80.00 & 96.00 & 80.00 \\
2 & $1-3$ & 44.00 & 52.80 & 44.00 \\
5 & $2-5$ & 44.00 & 52.80 & 44.00 \\
\hline
\end{tabular}

Cas $\mathrm{N}^{\circ} 2$

Dans ce cas, le coût désiré $C_{0}$ est inférieur au coût optimal du cas 1. Les resultants de test pour trois valeurs de coûts désirés, sont montrés ci-dessous

\section{$\checkmark$ Cas 2.1}

Avec: $C_{0}=840.00 \$ / \mathrm{h}$ et $\delta_{c 0}=61.00 \$ / \mathrm{h}$.

Solution: $\mathrm{z}=0.80$, Coût $=852.20 \$ / \mathrm{h}$, Pertes =5.61 MW.

$\checkmark$ Cas 2.2

Avec: $C_{0}=800.00 \$ / \mathrm{h}$ et $\delta_{c 0}=101.00 \$ / \mathrm{h}$.

Solution: $\mathrm{z}=0.603 \quad$ Coût $=840.09 \$ / \mathrm{h}$,

Pertes $=5.76 \mathrm{MW}$.

\section{$\checkmark \quad$ Cas 2.3}

Avec: $C_{0}=780.00 \$ / \mathrm{h}$ et $\delta_{c 0}=121.00 \$ / \mathrm{h}$.

Solution: $\mathrm{z}=0.530 \quad$ Coût $=836.51 \$ / \mathrm{h}$,

Pertes $=5.83 \mathrm{MW}$.

Les contraintes des puissances actives transmises, qui dépassent leurs limites normales, sont montrées dans la Figure I-2.

En comparant ces résultats avec ceux du cas 1, on remarque que le coût tend à baisser avec la diminution du coût désiré $C_{0}$. Cependant, les puissances transmises par les lignes $\mathrm{n}^{\circ} 1,2$ et 5 dépassent légèrement leurs limites normales (moins de 10\%). Les pertes de puissance active, ont aussi augmentées, avec la diminution de $C_{0}$

Le temps de convergence a été entre 2.11 et 2.31 secondes, et le processus a convergé à la 6ème itération. 
Il est clair que lorsqu'on réduit le coût désiré, l'algorithme FOPF, procède à l'équilibre de l'échange coût - surcharge des lignes. IL est. Important de souligner que si les surcharges des lignes 1, 2 et 5 sont acceptables (environ 9\%), le gain de $22.55 \$ / \mathrm{h}$ en coût de production, dans le cas 2.3 , serait très significatif.

Le coût de production baisse et les lignes de transport, deviennent surchargés au dessus de la valeur normale. Ceci traduit clairement les échanges (trade-off) qui existent entre le coût de production flous et les contraintes floues.

La valeur du nombre flou z (qui est la fonction d'appartenance composée à partir du minimum de toutes les fonctions d'appartenances des contraintes floues), dans le cas 2.1 est supérieure à celle du cas 2.2. Ceci montre que malgré que le coût dans le cas 2.2 soit plus faible, il est plus significatif ou il est plus juste d'utiliser la solution du cas 2.1, avec un degré de satisfaction meilleur $(\mathrm{z}=0.80)$.

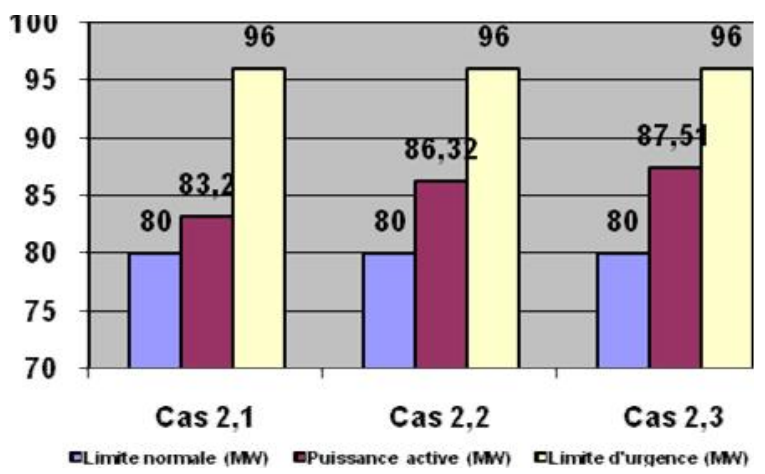

(a) Pour la ligne $\mathrm{N}^{\circ} 1$ entre les jeux de barres 1 et 2

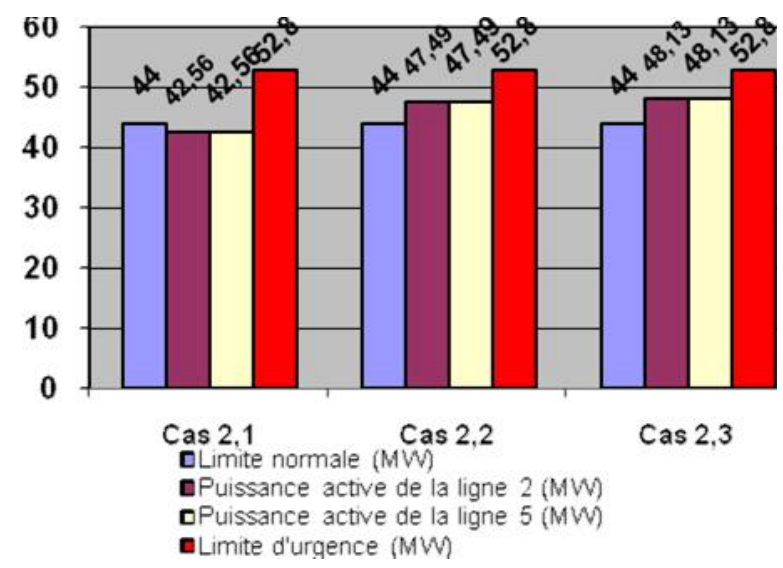

(b) (Pour les lignes $\mathrm{N}^{\circ} 2$ et $\mathrm{N}^{\circ} 5$ )

Fig.2. Puissances actives transmises dépassant leurs limites normales du réseau électrique à $30 \mathrm{~J} . \mathrm{B}$. - cas $\mathrm{n}^{\circ} 2$

Cas $n^{\circ} 3$ 
Ce cas est similaire au cas 2 , sauf que la limite d'urgence de la ligne $\mathrm{n}^{\circ} 1$ est réduite. C'est-àdire que $\delta_{d 1}$ est inférieur à celui du cas 2 . Les résultats de test pour les:

Cas 3.1

Avec: $C_{0}=840.00 \$ / \mathrm{h}$ et $\delta_{c 0}=61.00 \$ / \mathrm{h}$.

Solution: $\mathrm{z}=0.817$, Coût $=851.15 \$ / \mathrm{h}$, Pertes $=5.45 \mathrm{MW}$.

Cas 3.2

Avec: $C_{0}=800.00 \$ / \mathrm{h} \mathrm{et} \delta_{c 0}=101.00 \$ / \mathrm{h}$.

Solution $\mathrm{z}=0.5869$, Coût $=841.72 \$ / \mathrm{h}$, Pertes $=5.59 \mathrm{MW}$

$\checkmark$ Cas 3.3

Avec $C_{0}=780.00 \$ / \mathrm{h}$ et $\delta_{c 0}=121.00 \$ / \mathrm{h}$.

Solution $\mathrm{z}=0.513$, Coût $=838.87 \$ / \mathrm{h}$, Pertes $=5.66 \mathrm{MW}$

Le temps de convergence a été entre 2.40 et 2.77 secondes, et le processus a convergé à la 6ème itération.

On remarque que les coûts de production ont changés et ils sont supérieurs à ceux du cas 2 , et aussi d'après la Figure $(\mathrm{I}-3)$, la puissance active transmise par la ligne $\mathrm{n}^{\circ} 1$ est inférieure à celle du cas 2. Ceci s'explique par le fait que pour le même taux de surcharge, la fonction appartenance est plus faible dans le cas 3. Par conséquent, la contrainte de puissance active des lignes, dans ce cas, a plus de poids comparé au cas 2, dans le processus d'optimisation floue.

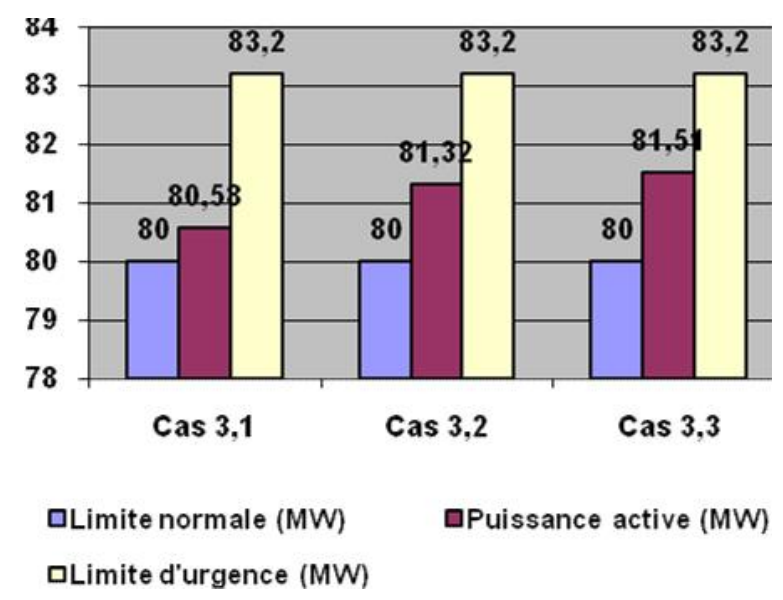

(a) Pour la ligne $\mathrm{N}^{\circ} 1$ entre les jeux de barres 1 et 2 


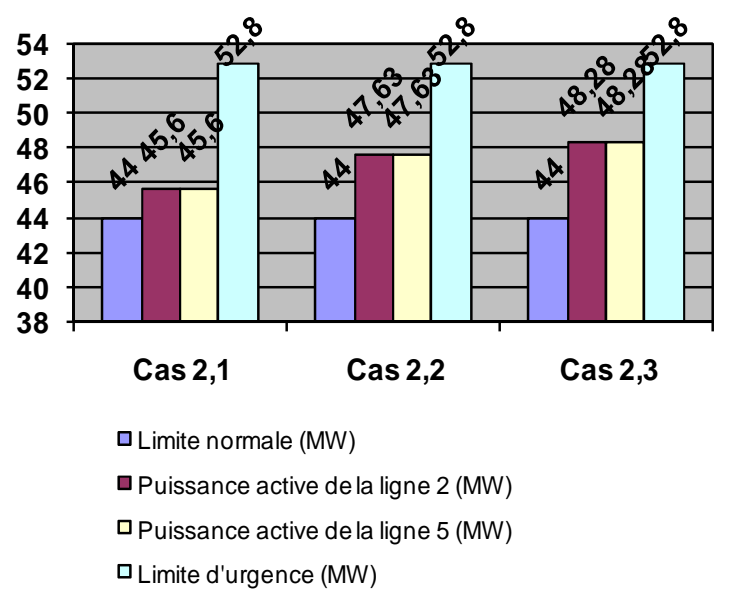

(b) Pour les lignes $\mathrm{N}^{\circ} 2$ et $\mathrm{N}^{\circ} 5$

Fig.3. Puissances actives transmises dépassant leurs limites normales du réseau électrique à $30 \mathrm{~J} . \mathrm{B} .-\operatorname{cas}^{\circ} 3$

\section{CONCLUSION}

Une application des ensembles flous au problème de la répartition optimale de puissance, a été exposée en détail. L'incertitude qui caractérise les contraintes de fonctionnement, ainsi que la fonction coût, ont été modélisées par des nombres flous de formes demi - trapézoïdales.

La méthodologie adoptée a été de convertir la formulation floue en une formulation conventionnelle, en utilisant les opérations de base des ensembles flous. Un algorithme basé sur la programmation linéaire successive (SLP), a été utilisé pour résoudre le nouveau problème obtenu.

\section{REFERENCES}

[1] Cui-Ru Wang, He-Jin Yuan, Zhi-Qiang Huang, Jiang-Wei Zhang, Chenjun Sun. “A modified particle swarm optimization algorithm and its application in optimal power flow problem," IEEE Proceeding of the fourth International Conference on machine. Learning and Cybernetics, Guangzhou, 18-21 August 2005

[2] K. Tomosovic, M.Y. Chow "Tutorial on Fuzzy Logic Applications in Power Systems", IEEE-PES, Winter Meeting in Singapore, January 2000.

[3] V. Miranda and J. T. Saraiva, "Fuzzy Modeling of P Flow," IEEE Transactions on Power Systems, Vol. 7, No. 2, May 1992, pp. 843-949. 
[4] W. Mielczarski, "Fuzzy Logic Techniques in Power Systems", New York, PhysicaVerlag Heidelberg, 1998.

[5] A. Soos, “An Optimal Adaptive Power System Stabilizer”, PHD. Thesis, University Of Calgary, October 1997

[6] K.H. Abdul-Rahman, and S.M. Shahidehpour , "Static Security in Power System Operation with Fuzzy Real Load Constraints," IEEE Trans. On Power Systems, Vol.10, No.1, 1995, pp77-87. ower System Optimal Load 


\section{APPLICATION DES ENSEMBLES FLOUS A LA REPARTITION \\ OPTIMALE DE LA PUISSANCE DANS LES RESEAUX ELECTRIQUES}

\section{RESUME}

Habituellement, le calcul de la répartition optimale de la puissance ou l'écoulement de puissance optimal (OPF), au niveau d'un réseau électrique, emploie des techniques de programmation mathématique standard. Parfois ces techniques ne sont pas convenables pour traiter certaines considérations pratiques rencontrées dans les systèmes de puissance, telle que l'incertitude des contraintes de fonctionnement [1].

Pour bien prendre en compte ce type de contraintes, on propose dans ce travail l'application d'une méthode basée sur les ensembles flous, au problème de la répartition optimale des puissances, connue aussi par «L'écoulement de puissance optimal »( $\mathrm{OPF})$. La méthode développée a été testée sur des réseaux électriques standards à moyenne échelle (30 J.B).

Mots clés : l'écoulement de puissance optimal, l'écoulement de puissance optimal par logique floue, Programmation mathématique de la logique floue.

How to cite this article

Guediri A and Ben Attous D. Application of fuzzy ensembles for optimal distribution of power in electrical networks. J Fundam Appl Sci. 2011, 3(1), 1-13. 